\title{
Study on Thyroid Profile In RDS
}

\author{
Dr. Antony Kisku ${ }^{1}$, Dr. Kiran Kumar M.N ${ }^{2}$, Dr. Deepak Chaturvedi ${ }^{3}$, \\ Dr. M R Akhouri ${ }^{4}$ \\ Postgraduate ${ }^{I 23}$, Associate professor ${ }^{4}$, Rajendra Institute Of Medical Sciences, Ranchi, Jharkhand
}

\begin{abstract}
Respiratory Distress syndrome(RDS) in newborn is defined as the presence of any two of the following features: 1. Respiratory rate >60. 2.Subcostal/Intercostal retractions. 3.Expiratory grunt/Groaning.In addition, presence of nasal flaring, suprasternalretractions, decreased air entry on auscultation of chest wall will also indicate the presence of respiratory distress. An infant who has an advanced degree of respiratory distress may exhibit additional signssuch as cyanosis, gasping,choking, apnea,stridor.It is more common in early and late pre-term babies.Thyroid function including total T3,T4,TSH were studied in these patients.50 premature new-born with respiratory distress syndrome(RDS),40 prematures comparable by gestational age who failed to develop RDS and 20 full term normal babies.The group of premature newborn infants in whom RDS occurred as compared to other two groups had significantly lower mean total T3,T4 level when matched by gestational age,the difference were also significant.Two days following delivery, the total serum T3,T4 level in the surviving prematures with RDS as compared with prematures without RDS was significantly lower. The mean value for Total T3,T4 and TSH in the respective mothers of the three groups were not significantly different.Since L-thyroxinehas been shown to increase the production of lung surfactant, widely accepted as deficient in RDS newborn infants, an association between lung immaturity and fetal thyroid function postulated.
\end{abstract}

Keywords: RDS, retraction, thyroid function, l-thyroxine

\section{Intoduction}

$\star$ Respiratory Distress Syndrome (RDS) in newborn is defined as the presence of any two of the following:

1. Respiratory rate $>=60 / \mathrm{min}$.

2.Subcostal/Intercostal retractions.

3.Expiratory grunt/Groaning. $\star$ In addition presence of suprasternal retractions, nasalflaring, decreased air entryon auscultation of the chest will also indicate respiratory distress.

$\star$ An infant who has advanced degree of RDS may also exhibit gasping, cyanosis, apnea, choking.

$\star$ RDS is common in pre-term and late pre-term newborns and is one of the most common cause of mortality in Nicu $(38 \%)$

$\star$ The following study has been done to see the variation in thyroid profile in pre-term newborns with RDS and those who failed to develop it.

$\star$ Previously too a lot of studies has been done in the past related to thyroid status in pre-terms but not a single study has been done in pre-terms with RDS.

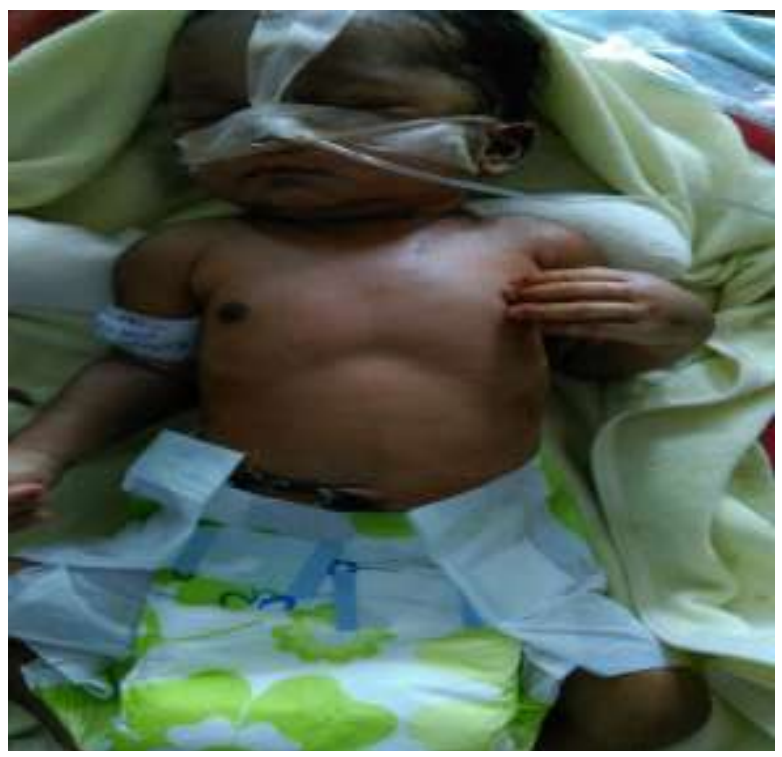




\section{Aims And Objectives}



1. To study thyroid profile (free T3, T4, TSH) in newborns with Respiratory Distress Syndrome (RDS).

2. To see its variations in pre-terms without RDS and normal term newborns.

3. To establish correlation of thyroid profile with surfactant deficiency

\section{Material And Methodology}

$\star$ Around 60 pre-term (early and late) newborns admitted in RIMS (Rajendra Institute of Medical Sciences) NICU and diagnosed to be RDS were included in the study.

$\star$ Among the controlled groups were the 40 pre-term newborns who failed to develop RDS and 20 normal term newborns.

$\star$ The thyroid profile (Free T3, T4and TSH) were studied in all the cases and controlled groups and were compared.

$\star$ Even the thyroid profile of their respective mothers were obtained

to ensure that they did not have any thyroid related disorders.

\section{Inclusion criterias:}

$\star$ All the pre-term newborns with following features were included in the study:

1. Respiratory rate $>=60 / \mathrm{min}$.

2. Subcostal/Intercostal retractions.

3. Expiratory grunting/Groaning.

4. Cyanosis, Gasping, choking, apnea, stridor.

5. Nasal flaring.

\section{Methodology}

After aseptic precautions blood was obtained from all the above newborns and were sent for thyroid profile study only after theirs first 48 hours of life i.e., $3^{\text {rd }}$ day of life onwards. Even the thyroid profile of their respective mothers were obtained.

\section{Results}

$\star$ The following results were obtained based on the study




Table: Showing The Value Of Free T4 In Pre-Term Newborn With Rds

\begin{tabular}{|l|l|l|l|l|}
\hline FREE T4 (ng/dl) & $<0.3$ & $0.3-0.6$ & $0.6-0.7$ & $0.7-5.5$ \\
\hline NO. OF PTS. & 45 & 9 & 4 & 2 \\
\hline
\end{tabular}

Maximum pre-term with RDS (45) had their free T4 level in the very low range. The normal value being taken 0.7-5.5ng/dl

Table: Showing Free T3 In Pre-Term Newborns With Rds

\begin{tabular}{|l|l|l|l|l|}
\hline FREE T3 (ng/dl) & $<0.5$ & $0.5-0.10$ & $0.10-0.15$ & $0.15-2.0$ \\
\hline NO. OF PTS. & 32 & 15 & 9 & 4 \\
\hline
\end{tabular}

Maximum pre-term with RDS (32) had their free T3 level in the very low range. The normal value being taken as $\mathbf{0 . 1 5 - 2 n g / d l}$

Table: Showing Tsh Level In Pre-Term Newborns With Rds

\begin{tabular}{|l|l|l|l|l|}
\hline TSH(mu/l) & $<0.1$ & $0.1-0.3$ & $0.3-0.5$ & $0.5-15$ \\
\hline NO. OF PTS. & 1 & 2 & 7 & 50 \\
\hline
\end{tabular}

50 Pre-term newborns with RDS had their TSH level in the normal level (0.5-15 mu/L)

Table: Showing Free T4 In Pre-Term Newborns Who Did Not Develop Rds

\begin{tabular}{|l|l|l|l|l|}
\hline FREE T4 (ng/dl) & $<0.3$ & $0.3-0.6$ & $0.6-0.7$ & $0.7-5.5$ \\
\hline NO. OF PTS. & 3 & 4 & 18 & 15 \\
\hline
\end{tabular}

Maximum Pre-term who did not develop RDS (18)had their Free T4 level in slightly lower level (0.6-0.7 ng/dl).

Table: Showing Free T3 In Pre-Term Newborns Who Did Not Develop Rds

\begin{tabular}{|l|l|l|l|l|}
\hline FREE T3(ng/dl) & $<0.5$ & $0.5-0.10$ & $0.10-0.15$ & $0.15-2.0$ \\
\hline NO. OF PTS. & 4 & 3 & 12 & 21 \\
\hline
\end{tabular}

Maximum Pre-term who did not develop RDS (21) had their Free T3 level in the normal range (0.15-2 ng/dl)

Table: Showing Tsh Level In Pre-Term Newborns Who Did Not Develop Rds

\begin{tabular}{|l|l|l|l|l|}
\hline TSH(mu/l) & $<0.1$ & $0.1-0.3$ & $0.3-0.5$ & $0.5-15$ \\
\hline NO.OF PTS. & 1 & 3 & 2 & 34 \\
\hline
\end{tabular}

34 Pre-term who did not develop RDS had their TSH level in the normal range $\mathbf{( 0 . 5 - 1 5 m u / l )}$

Out of $\mathbf{2 0}$ normal term newborns only $\mathbf{2}$ had slightly lower level of Free T4 level and $\mathbf{1 8}$ of them had normal thyroid profile.



\section{Discussion}

As per the study done it could be quite clearly seen that out of 60 pre-term newborns who developed RDS nearly everyone had low value of free T4 and T3 levels. As RDS is a very common cause for neonatal mortality in our setting and surfactant being not affordable it is the need of the hour to have some less economical replacement for it. Even Ralph et al in their study in 1974 showed that out of 34 pre-term newborn with RDS, 27 babies had their mean Free T4 level in the very lower range below the normal value. 


\section{Limitation Of The Study}

The number of cases selected were not adequate.

The pre-term newborns who developed RDS had their mean gestational age lower than those pre-terms who failed to develop it.

\section{Conclusion}

The Free T3 and T4 was very much lower in almost every cases of Pre-term newborn who developed RDS. The normal value of free T3 being taken as (0.15-2 ng/dl) and that of freeT4 being taken as $(\mathbf{0 . 7 - 5 . 5} \mathbf{~ g / d l )}$ Out of 40 pre-terms who did not develop RDS 19cases had their mean Free T3 and25 cases had their Free T4 level in the lower range. TSH level was nearly normal in both the above groups. With the above study a correlation can be established between the surfactant deficiency and Free T3 and T4 levels in RDS in newborns and thyroxine can turn out to be replacement of surfactant in the near future. Now as per the limitations being mentioned above a lot of studies are still required to establish a correlation between thyroid deficiency and surfactant deficiency in newborn with RDS.

\section{Bibliography}

[1]. Nelson text book pf paediatrics $20^{\text {th }}$ edition

[2]. Cloherty text book $f$ newborn care $7^{\text {th }}$ edition

[3]. Avery's newborn

[4]. Ralph study on RDS (1974) 